\title{
On stochastic stability of regional ocean models with uncertainty in wind forcing
}

\author{
L. M. Ivanov and P. C. Chu \\ Naval Postgraduate School, Monterey, CA, USA
}

Received: 21 November 2006 - Revised: 10 April 2007 - Accepted: 9 October 2007 - Published: 25 October 2007

\begin{abstract}
A shallow-water model was used to understand model error induced by non-Gaussian wind uncertainty. Although the model was simple, it described a generic system with many degrees of freedom randomized by external noise. The study focused on the nontrivial collective behavior of finite-amplitude perturbations on different scales and their influence on model predictability. The error growth strongly depended on the intensity and degree of spatial inhomogeneity of wind perturbations. For moderate but highly inhomogeneous winds, the error grew as a power law. This behavior was a consequence of varying local characteristic exponents and nonlinear interactions between different scales. Coherent growth of perturbations was obtained for different scales at various stages of error evolution. For the nonlinear stage, statistics of prediction error could be approximated by a Weibull distribution. An approach based on the Kullback-Leibler distance (the relative entropy) and probability-weighted moments was developed for identification of Weibull statistics. Bifurcations of the variance, skewness and kurtosis of the irreversible predictability time (a measure of model prediction skill) were detected when the accepted prediction accuracy (tolerance) exceeded some threshold.
\end{abstract}

\section{Introduction}

When circulation is simulated by a fine resolution regional model in an area with open boundaries, the circulation dynamics often depends crucially on specified open boundary conditions, wind forcing and sub-scale parameterizations. For atmospheric predictability, it is generally assumed that the model forecasting is most sensitive to uncertainty of initial conditions. However, for oceanic predictability in

Correspondence to: L. M. Ivanov

(lmivanov@nps.edu) marginal seas with lengthy coastal zones, impact of uncertainty of external forcing and/or subgrid parameterizations, may be as significant as errors in initial conditions. The model blow-up often sets in much faster than the model loses its predictability due to errors in initial conditions (Jiang and Malanotte-Rizzoli, 1999; Boffetta et al., 2000; Bogden, 2001; Auclair et al., 2003, among others).

Starting from the pioneering work of Lorenz (1963), the dynamics of the prediction error (PE) due to uncertainty in initial conditions has been deeply investigated in the theoretical and numerical studies. The dynamics of model-related errors has been paid much less attention to, probably due to the large variety of possible modeling errors. Although some general trends have emerged (Capotondi and Holland, 1997; Chu et al., 1999; Orrell et al., 2001; Vannitsem and Toth, 2002; Nicolis, 2003, 2004, among others), more refined theoretical investigations and additional experiments with an hierarchy of ocean models of different levels of complexity are necessary to get a more general view of the impact of model-related error (particularly finite-amplitude) on model predictability.

In the present paper, the dynamics of a model-related error (hereafter, prediction error (PE)) generated by uncertainty in wind forcing and its impact on predictability are studied in the context of stochastic model stability (stability answered in terms of probabilistic measures, such as expected values or distribution functions (Freidlin and Wentzell, 1998)) for a simplified regional model destabilized near an unstable equilibrium state (an unstable fixed point in model phase space) by stochastic wind.

In general, the stochastic stability and predictability differ from one another. However, if a time scale quantifies the model predictability, and if this scale indicates the time when the forecast uncertainty exceeds some boundary or when information on the initial condition is lost, the stochastic stability and predictability are interchangeable. Since such time scales are widely used in meteorology (see, for example,

Published by Copernicus Publications on behalf of the European Geosciences Union and the American Geophysical Union. 
Toth, 1991) and oceanography (Robinson et al., 1996), the stochastic stability concept seems to be a useful tool for the predictability analysis of large ocean models, and "the irreversible predictability time" (a prediction time scale) (Chu et al., 2002) is a quantitative measure of model predictability.

Numerical computations discussed below can have a twofold interpretation. First, the obtained results can be interpreted as stability of a circulation regime relative to a highfrequency component of wind forcing. Second, if we parameterize uncertainty of wind forcing as a stochastic noise, the computation results are interpretable in the predictability context. Therefore, hereafter, perturbations excited by stochastic wind in an ocean basin will also be called a prediction error.

The principal motivation of the proposed study is formulated as follows.

First, it is well-known from numerical modeling that wind is one of the main energy sources of ocean currents and that, naturally, prediction errors associated with wind forcing uncertainty may grow quickly in numerical models (Robinson et al., 1996; Berloff and McWilliams, 1999; Chu et al., 1999; Sura et al., 2001; Burillo et al., 2002, and others). Behavior of such model-related errors are highly dependent on the properties of the underlying dynamical regime [attractor] reproduced by the numerical model and statistics of wind uncertainty. Circulation patterns with oscillations near quasiequilibrium states and transition dynamics between them are typical for many marginal seas, such as the Black Sea (Stanev and Staneva, 2000), and for large-scale jet-like currents like Kuroshio (Masuda et al., 1999). The proposed study focuses on the case of evolution of finite-amplitude non-Gaussian perturbations induced by stochastic wind error when the ocean is near a quasi-stable equilibrium state (even small perturbations can destroy such a state and stimulate a transition to another one). Therefore, the obtained results are important for understanding the regional model predictability (Robinson et al., 1996) when local attractor features determine the phase-spatial organization of the local error growth rate.

Second, in ocean models, unresolved dynamics is often represented in terms of random forcing. For example, impact of mesoscale eddies on large-scale currents can be approximated as a space-time correlated, random-forcing process (Berloff, 2005). Therefore, the results obtained in the present study may be useful for interpretation of a wide spectrum of problems related to model predictability in the atmosphere and ocean.

The rest of the paper is organized as follows. Section 2 explains a predictability metrics used to quantify the model predictability for both small- and large-amplitude perturbations. Features of the reference solution (the control run) are discussed in Sect. 3. The surface wind is decomposed into two parts: steady ("climatic") part and stochastic one caused by unknown synoptic variability. Statistics of the wind uncertainty is given in Sect. 4. The model phase space is intro- duced in Sect. 5. Section 6 investigates the sensitivity of the root mean square prediction error to variations of stochastic wind and the tolerance level (the accepted prediction accuracy). Section 7 analyzes finite-amplitude induced phase transitions of predictability. Section 8 develops a technique for identification of the probability density function of the irreversible predictability time. Herein, we demonstrate that the statistics of this time is rather Weibullian than Gaussian. The predictability horizon is estimated in Sect. 9. Section 10 provides the conclusions. Appendix A contains analytical representations for the wind error source term.

\section{Predictability measures}

We examine the sensitivity of a reference solution relative to stochastic variations of wind forcing. Such sensitivity may be measured by the traditional non-dimension root mean square difference between perturbed ("pert") and nonperturbed ("ref") solution presented by a variable $\Psi$, which may stand for energy, temperature, salinity, the stream function etc. (Holland and Malanotte-Rizzoli, 1989; Brasseur et al., 1996; Robinson et al., 1996; Wirth and Ghil, 2000, among others),

$$
<\bar{I}(t)>=<I_{\text {pert }}^{2}(t)>/ I_{\text {ref }}^{2}(t)
$$

and the irreversible predictability time (IPT) (Ivanov et al., 1994) defined as

$\tau(\bar{\varepsilon})=\inf _{t \geq 0}\left(t \mid \bar{I}(t)>\bar{\varepsilon}^{2}\right)$,

where $I_{\text {pert }}=\left\|\Psi_{\text {pert }}-\Psi_{\text {ref }}\right\|, I_{\text {ref }}=\left\|\Psi_{\text {ref }}\right\|$, hereafter $<\ldots>$ is the ensemble averaging, $\bar{\varepsilon}=\varepsilon / I_{\text {ref }}$ is the non-dimension tolerance (the accepted prediction accuracy), \|\| is the Euclidean norm. According to Eq. (2) the irreversible predictability time is a time at which the prediction error $\bar{I}^{1 / 2}$ reaches a predetermined level $\bar{\varepsilon}$ for the first time, i.e. any returns of model predictability are impossible after $\tau(\bar{\varepsilon})$.

The IPT is clearly interpretable in a model phase space, where perturbed and non-perturbed (reference) solutions are represented by $\boldsymbol{X}^{\prime}$ and $\boldsymbol{X}$ trajectories, respectively, and the equation $\bar{I}(t)=\bar{\varepsilon}^{2}$ describes a spheroidal surface $S(\bar{\varepsilon})$ moving along the trajectory $\boldsymbol{X}$ (Fig. 1a). A distance $\left|\boldsymbol{X}_{o}-\boldsymbol{X}_{o}^{\prime}\right|$ between the trajectories at time moment $t_{o}$ (the initial error) usually grows with time due to model inaccuracy, and becomes larger than $\bar{\varepsilon}$ (crossing $S(\bar{\varepsilon})$ ) after a time $\tau(\bar{\varepsilon})$ (Fig. 1a). This time is defined as the IPT.

For a steady reference solution (represented by a fixed point in the model phase space), the IPT becomes the classical first passage time (FPT). The classical FPT is a time at which a trajectory reaches a boundary for the first time (Gardiner, 1985). Therefore, the IPT can also be defined as the FPT for varying boundaries (compare Fig. 1a and Fig. 1b). The FPT plays an essential role in many applied fields. We 


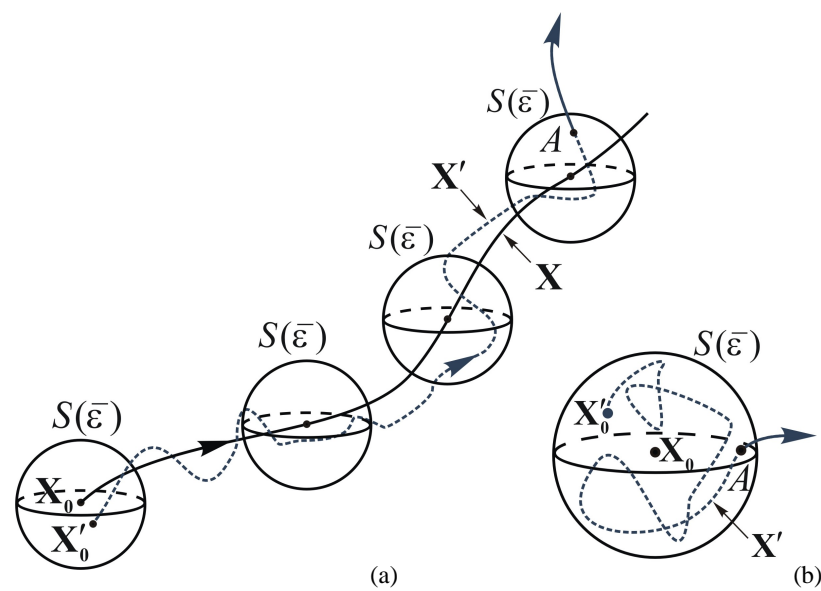

Fig. 1. Definitions of (a) IPT and (b) FTP. Phase trajectories $\boldsymbol{X}$ (the reference solution) with the initial position of $\boldsymbol{X}_{0}$, and $\boldsymbol{X}^{\prime}$ (a perturbed solution) with the initial position of $\boldsymbol{X}_{0}^{\prime}$ are denoted by solid and dashed curves, respectively. A time when $\boldsymbol{X}^{\prime}$ crosses $S(\bar{\varepsilon})$ in the point $\mathrm{A}$ is the IPT (a) or FPT (b).

can suppose that the IPT may become a useful tool for the analysis of ocean model predictability too.

Statistics of the IPT (Ivanov et al., 1994; Chu and Ivanov, 2005) can be represented by the probability density function $(\tau$-PDF) or the cumulative distribution function $(\tau-\mathrm{CDF})$ $\left[P\left(\bar{\varepsilon}, t-t_{0}\right)\right] . \quad \tau$-CDF is the probability that $\tau \geq t-t_{0}$ for a given tolerance level.

In practical applications, statistics of IPT ( $\tau$-CDF or $\tau$ PDF) may be identified from $\tau$-moments,

$\tau_{l}(\bar{\varepsilon})=l \int_{t_{0}}^{\infty}\left(t-t_{0}\right)^{l-1} P\left(\bar{\varepsilon}, t-t_{0}\right) d t, \quad l=1, \ldots, L$.

Knowing these moments we may compute $\tau$-mean , $\tau$ variance $(\tau$-var),$\tau$-skewness ( $\tau$-sk) and $\tau$-kurtosis $(\tau$-ku). For example,

$\tau$-mean $=\tau_{1}, \tau$-var $=\tau_{2}-\left(\tau_{1}\right)^{2}$ etc.

If the cumulative distribution function $\mathrm{P}$ has a heavy tail for large values of $t-t_{0}$, high-order $\tau$-moments $(l=2, \ldots, L)$ sometimes do not exist because the integral in Eq. (3) does not converge. In this case we suggested (Ivanov and Chu, $2007)$ to identify $\tau$-CDF or $\tau$-PDF from the probabilityweighted moments $\left(\alpha_{l}\right)$ defined originally by Greenwood et al. (1979),

$\alpha_{l}=\int_{0}^{1} X(P)(1-P)^{l} d P, \quad l=1, \ldots, L$,

where $X(P)$ is the quantile function (i.e., the inverse of cumulative distribution function).

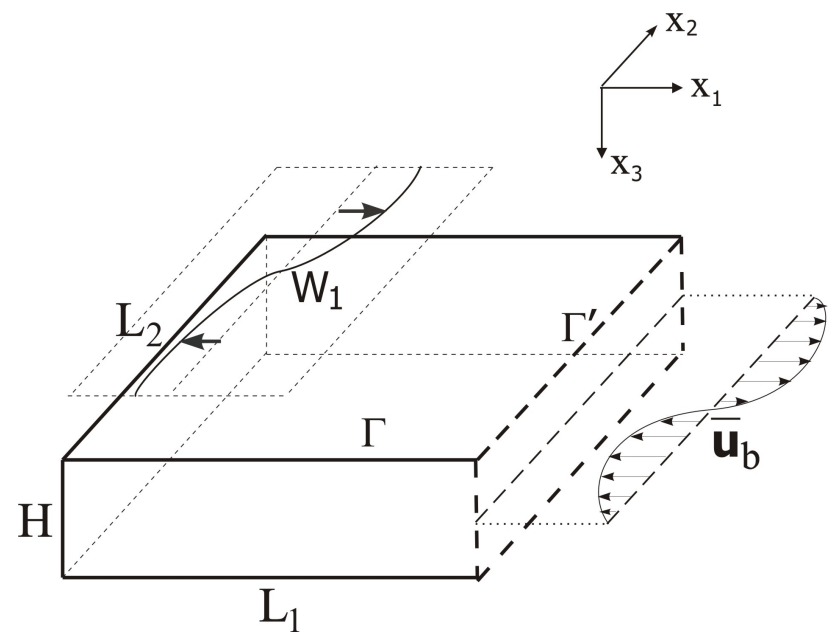

Fig. 2. Basin geometry. The $x_{1}$ and $x_{2}$ axes point toward east and north, respectively.

In practice the same moments $\alpha_{l}$ are estimated from an ordered random sample $(\tau)_{n=1}^{N}$ of size $N$ (Hosking and Wallis, 1997) by

$\hat{\alpha}_{l}=\frac{1}{N}\left(C_{l}^{N-l}\right)^{-1} \sum_{n=1}^{N} C_{l}^{n-l}(\tau)_{n}^{N}$,

where $C_{l}^{n-l}$ are the binomial coefficients.

The probability-weighted moments always exist and are robust relative to sampling error. Therefore, the robust estimate of $\tau$-CDF or $\tau$-PDF for small forecast ensembles is possible. This is one of advantages of the IPT. An appropriate method for estimating distribution functions from knowledge of the probability weighted moments will be discussed in Sect. 8.

The moments of IPT as functions of $\boldsymbol{X}_{o}-\boldsymbol{X}_{o}^{\prime}$ satisfy the Pontryagin-Kolmogorov-Stratonovich equations (Pontryagin et al., 1969), which are linear elliptic differential equations. Their asymptotic solutions can be obtained in many cases. For example, Chu et al. (2002) calculated analytically first two moments of IPT for a low-order nonlinear atmospheric model (Lorenz, 1984). Therefore, the analytical estimate of model predictability in the IPT context is another advantage of our approach.

\section{The reference solution}

We consider a rectangular semi-closed basin with the horizontal dimensions: $L_{1}=1050 \mathrm{~km}$ and $L_{2}=1000 \mathrm{~km}$, and with constant depth $H=2 \mathrm{~km}$, which is situated on a mid-latitude $\beta$-plane. The basin has rigid $(\Gamma)$ and open $\left(\Gamma^{\prime}\right)$ boundaries. The geometry of the basin and its sizes are shown in Fig. 2 . 
Our numerical model is the nonlinear shallow-water equations with nonlinear bottom friction, wind and boundary forcing

$$
\begin{aligned}
& \frac{\partial D u_{1}}{\partial t}+L\left(D u_{2}, D u_{1}\right)-f D u_{2}=-g D \nabla_{1} \zeta+W_{1}-\alpha E^{1 / 2} u_{1}, \\
& \frac{\partial D u_{2}}{\partial t}+L\left(D u_{1}, D u_{2}\right)+f D u_{1}=-g D \nabla_{2} \zeta+W_{2}-\alpha E^{1 / 2} u_{2},
\end{aligned}
$$

and the mass conservation equation

$$
\frac{\partial \zeta}{\partial t}+\left(\nabla_{1} D u_{1}+\nabla_{2} D u_{2}\right)=0
$$

where $L(\ldots, \ldots)$ is the nonlinear advective operator; $\left[\nabla_{1}, \nabla_{2}\right]=\left[\frac{\partial}{\partial x_{1}}, \frac{\partial}{\partial x_{2}}\right] ; u_{1}$ and $u_{2}$ are the zonal and meridional velocities, respectively; $D=H+\zeta, \zeta$ is the sea surface elevation; the drag coefficient $\alpha=2.5 \times 10^{-3}$; the gravity $g$; and $E=u_{1}^{2}+u_{2}^{2}$.

The Coriolis parameter varies linearly with a beta plane approximation $f=f_{0}+\beta x_{2}$, where $f_{0}=2 \Omega \sin \left(\varphi_{o}\right)$ and $\beta=(2 \Omega / a) \cos \left(\varphi_{o}\right)$. Here, $\Omega$ and $a$ are the rate of rotation and the radius of the Earth, respectively; $\varphi_{0}=35^{\circ}$. For the chosen model parameters: $f_{o}=7.3 \times 10^{-5} \mathrm{~s}^{-1}$, $\beta=2.0 \times 10^{-11} \mathrm{~m}^{-1} \mathrm{~s}^{-1}$.

A flow in the semi-closed basin bounded by $\Gamma \cup \Gamma^{\prime}$ is forced by both the zonal wind forcing $W_{1}\left(W_{2}=0\right)$ varying with latitude as

$W_{1}=-\frac{w_{s}}{\rho_{w}} \cos \left(\frac{\pi x_{2}}{L_{2}}\right)$,

where $\rho_{w}=1025 \mathrm{~kg} \mathrm{~m}^{-3}, \quad w_{s}$ is the wind stress, $\frac{w_{s}}{\rho_{w}}=1.0 \times 10^{-3} \mathrm{~m}^{2} \mathrm{~s}^{-2}$, and a prescribed net flux (characterized by the normal velocity $\bar{u}_{b}\left(x_{2}, t\right)$ and surface elevation $\bar{\zeta}_{b}\left(x_{2}, t\right)$ along the boundary $\left.\Gamma^{\prime}\right)$. Zero normal velocity and zero Neumann conditions for the surface elevation are imposed on the rigid boundary $\Gamma$.

The chosen model configuration is suitable for the analysis of ocean model predictability affected by different kinds of stochastic uncertainties: errors inserted in initial conditions (Ivanov and Chu, 2007), wind (the present study) and open boundary conditions ${ }^{1}$. Cross-correlations between these errors can also be studied.

Model (6-8) is similar to that used by Veronis (1966) for the analysis of nonlinear wind-driven circulation in a closed basin. But in contrast to Veronis (1966) we parameterize bottom friction by the quadratic drag law (Pedlosky, 1987).

The barotropic mode of Princeton Oceanographic Model (Blumberg and Mellor, 1987) was applied to Eqs. (6-8) with the following model parameters: spatial resolution $-50 \mathrm{~km}$; time step -2 min.

The prescribed non-stationary net flux across the open boundary is computed as it was explained in Chu et

\footnotetext{
${ }^{1}$ Ivanov, L. M. and Chu, P. C.: Effects of stochastic open boundary uncertainty on predictability of regional ocean models, Mon. Weather Rev., in preparation, 2007.)
}

al. (1997). The structure of open boundary conditions on day- 0 and day-60 is demonstrated in Figs. $3 a$ and b, respectively. The initial condition represents a non-closed anticyclonic gyre shown in Fig. 3a. The corresponding initial surface elevation is not shown because its structure is obvious.

After 30 days of integration the model reaches a spin up when the spatially averaged kinetic energy oscillates with a period of 120 days. Amplitude of this oscillation reduces with time exponentially with rate of $1000 \mathrm{day}^{-1}$. The spatially averaged kinetic energy for the first 60 days is shown in Fig. 4a only because this time period is used for sensitivity studies. The circulation pattern formed after day-30 presents a multi-gyre structure with maximum velocities up to $0.9 . .1 .0 \mathrm{~m} / \mathrm{s}$ (Fig. 3b) and high surface elevation near $1 \mathrm{~m}$ (not shown).

\section{Wind forcing uncertainty}

Governing Eqs. (6-8) are perturbed by adding the stochastic wind forcing $\boldsymbol{w}=\left(w_{1}, w_{2}\right)$ to $\boldsymbol{W}=\left(W_{1}, W_{2}\right)$. The stochastic wind forcing is traditionally parameterized in the following form

$\boldsymbol{w}\left(x_{1}, x_{2}, t\right)=\frac{\rho_{\mathrm{air}}}{\rho_{w}} C_{d}\left|\boldsymbol{U}\left(x_{1}, x_{2}, t\right)\right| \boldsymbol{U}\left(x_{1}, x_{2}, t\right)$,

where $\rho_{\text {air }}$ is the air density $\left(1.3 \mathrm{~kg} \mathrm{~m}^{-3}\right), C_{d}\left(2 \times 10^{-3}\right)$ is the drag coefficient, and $\boldsymbol{U}$ is the stochastic wind.

Following Sura et al. (2001) $\boldsymbol{U}$ is represented by

$\boldsymbol{U}=\left[U_{1}\left(x_{1}, x_{2}, t\right), U_{2}\left(x_{1}, x_{2}, t\right),\right]=\boldsymbol{\mu}(t) \sigma G^{1 / 2}\left(x_{1}, x_{2}\right)$,

where $\boldsymbol{\mu}(t)=\left[\mu_{1}(t), \mu_{2}(t)\right]$ are white Gaussian vector processes with zero mean and unit variance; $\sigma^{2}$ is the wind variance; the spatial structure function $\mathrm{G}$ characterizes a degree of spatial inhomogeneity of wind perturbations above an area of interest.

Two different structure functions $G$ are used. The first one is given by

$G_{1}\left(x_{1}, x_{2}\right)=\cos \left(\frac{\pi x_{2}}{L_{2}}\right)$.

In this case only the amplitude of wind stress (Eq. 9) is distorted by the non-Gaussian white noise.

The second one is chosen as

$$
\begin{aligned}
G_{2}\left(x_{1}, x_{2}\right)= & \alpha_{\text {scale }}\left[\pi \beta_{1} \beta_{2} \operatorname{erf}\left(\frac{L_{1}}{2 \beta_{1}}\right) \operatorname{erf}\left(\frac{L_{2}}{2 \beta_{2}}\right)\right]^{-1 / 2} \\
& \exp \left(-\frac{\left(x_{1}-L_{1} / 2\right)^{2}}{2 \beta_{1}^{2}}-\frac{\left(x_{2}-L_{2} / 2\right)^{2}}{2 \beta_{2}^{2}}\right) .
\end{aligned}
$$

Here, erf is the error function; $\alpha_{\text {scale }}$ is a scaling parameter; $\left(\beta_{1}, \beta_{2}\right)$ are the decorrelation scales; $G_{2}$ shows the impact of the localized atmospheric eddy activity near the point $\left(L_{1} / 2, L_{2} / 2\right)$ on the surface wind perturbations (Sura et al., 2001). 

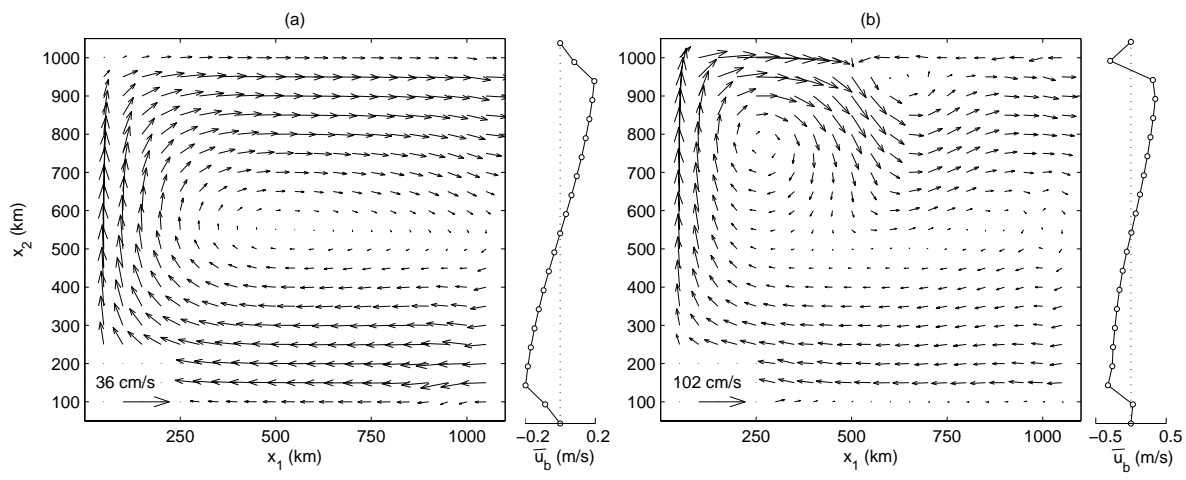

Fig. 3. Spatial structure of the reference solution at the initial state (a) and after integration for 60 days (b). Open boundary conditions $\left(\bar{u}_{b}\right)$ corresponding to the reference solution are shown to the right of the circulation patterns.

In most numerical experiments the scaling constant $\alpha_{\text {scale }}$ is chosen to adjust the weight function in Eq. (13) to 1 for $\beta_{c}=\beta_{1}=\beta_{2}=600 \mathrm{~km}$. However, a number of computations use $\beta_{c}$ between $100 \mathrm{~km}$ and $600 \mathrm{~km}$.

The noise in the surface wind with $\sigma^{2}=28.0 \mathrm{~m}^{2} \mathrm{~s}^{-2}$ corresponds to typical observed atmospheric conditions in the North Atlantic region (Wright, 1988). Therefore, the stochastic forcing (Eqs. 10-13) is a conceptual tool to study the effect of noise on simple and more complex wind-driven regional ocean models.

To understand statistics of wind forcing, Eq. (10) is rewritten into

$$
\begin{aligned}
\boldsymbol{w}\left(x_{1}, x_{2}, t\right) & =\frac{\rho_{\text {air }}}{\rho_{w}} C_{d} \sigma^{2} G\left(x_{1}, x_{2}\right)|\boldsymbol{\mu}(t)| \boldsymbol{\mu}(t) \\
& =\frac{\rho_{\text {air }}}{\rho_{w}} C_{d} \sigma^{2} G\left(x_{1}, x_{2}\right) \tilde{\boldsymbol{w}}
\end{aligned}
$$

where $\tilde{\boldsymbol{w}}=|\boldsymbol{\mu}(t)| \boldsymbol{\mu}(t)$. Then, the probability density function $f\left(\tilde{w}_{1}, \tilde{w}_{2}\right)$ is calculated using the elementary zero-memory transformations, which are discussed in most textbooks of probability theory (see, for example, Stratonovich, 1963). Accordingly to the general theory

$$
f\left(\tilde{w}_{1}, \tilde{w}_{2}\right)=f\left[g_{1}^{-1}\left(\tilde{w}_{1}, \tilde{w}_{2}\right), g_{2}^{-1}\left(\tilde{w}_{1}, \tilde{w}_{2}\right)\right] \cdot|J|,
$$

where $J$ is the Jacobian of the transformation from the random variables $\mu_{1}$ and $\mu_{2}$ to the random variables $\tilde{w}_{1}$ and $\tilde{w}_{2}$; $g_{1}^{-1}$ and $g_{2}^{-1}$ are the inverse functions.

Simple calculations result into

$$
f\left(\tilde{w}_{1}, \tilde{w}_{2}\right)=\frac{1}{4 \pi\left(\tilde{w}_{1}^{2}+\tilde{w}_{2}^{2}\right)^{1 / 2}} \exp \left[-\left(\tilde{w}_{1}^{2}+\tilde{w}_{2}^{2}\right)^{1 / 2} / 2\right] .
$$

The means $\left\langle\tilde{w}_{1}\right\rangle,\left\langle\tilde{w}_{2}\right\rangle$ and variances $\tilde{\sigma}_{1}^{2}, \tilde{\sigma}_{2}^{2}$ computed from Eq. (16) have the following values

$$
\left\langle\tilde{w}_{1}\right\rangle=\left\langle\tilde{w}_{2}\right\rangle=0.0 \text { and } \tilde{\sigma}_{1}^{2}=\tilde{\sigma}_{2}^{2}=3.0 \text {. }
$$

Both $\tilde{w}_{1}(t)$ and $\tilde{w}_{2}(t)$ are delta-correlated processes (Klyatskin, 2005).
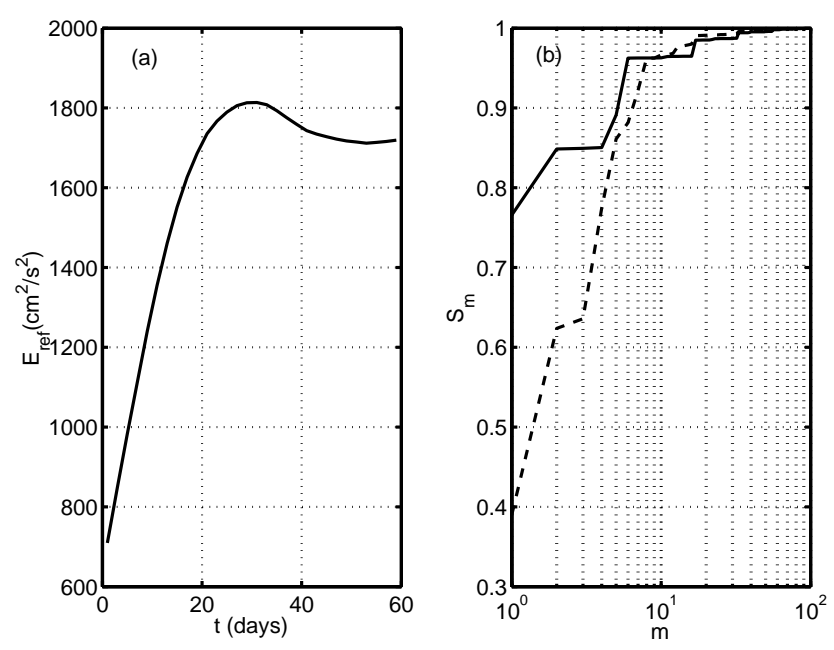

Fig. 4. Characteristics of the reference solution: (a) the kinetic energy averaged over the semi-closed basin, (b) the relative variance $S_{m}$ computed for the initial state of the reference solution (solid curve) and after integration for 60 days (dashed curve).

In the polar coordinate system $\{z, \theta\}\left[\tilde{w}_{1}=z \cos (\theta)\right.$, $\tilde{w}_{2}=z \sin (\theta)$ ], probability density function (Eq. 16) transforms to the following form:

$f(z, \theta)=\frac{1}{4 \pi} \exp (-z / 2)$.

Two-dimensional distribution Eq. (18) is easily transformed to a one-dimension form $f_{\text {int }}$ simply by integrating $f(z, \theta)$ with respect to $\theta$,

$f_{\text {int }}(z)=\frac{1}{2} \exp (-z / 2)$,

which is the exponential distribution.

The above calculations clearly indicate that although $\boldsymbol{U}$ is a white Gaussian process, the wind stress $\boldsymbol{w}$ is not. Therefore $\mathrm{PE}$ has non-Gaussian statistics even if the wind uncertainty is small. For large values of stochastic wind stress, distribution 

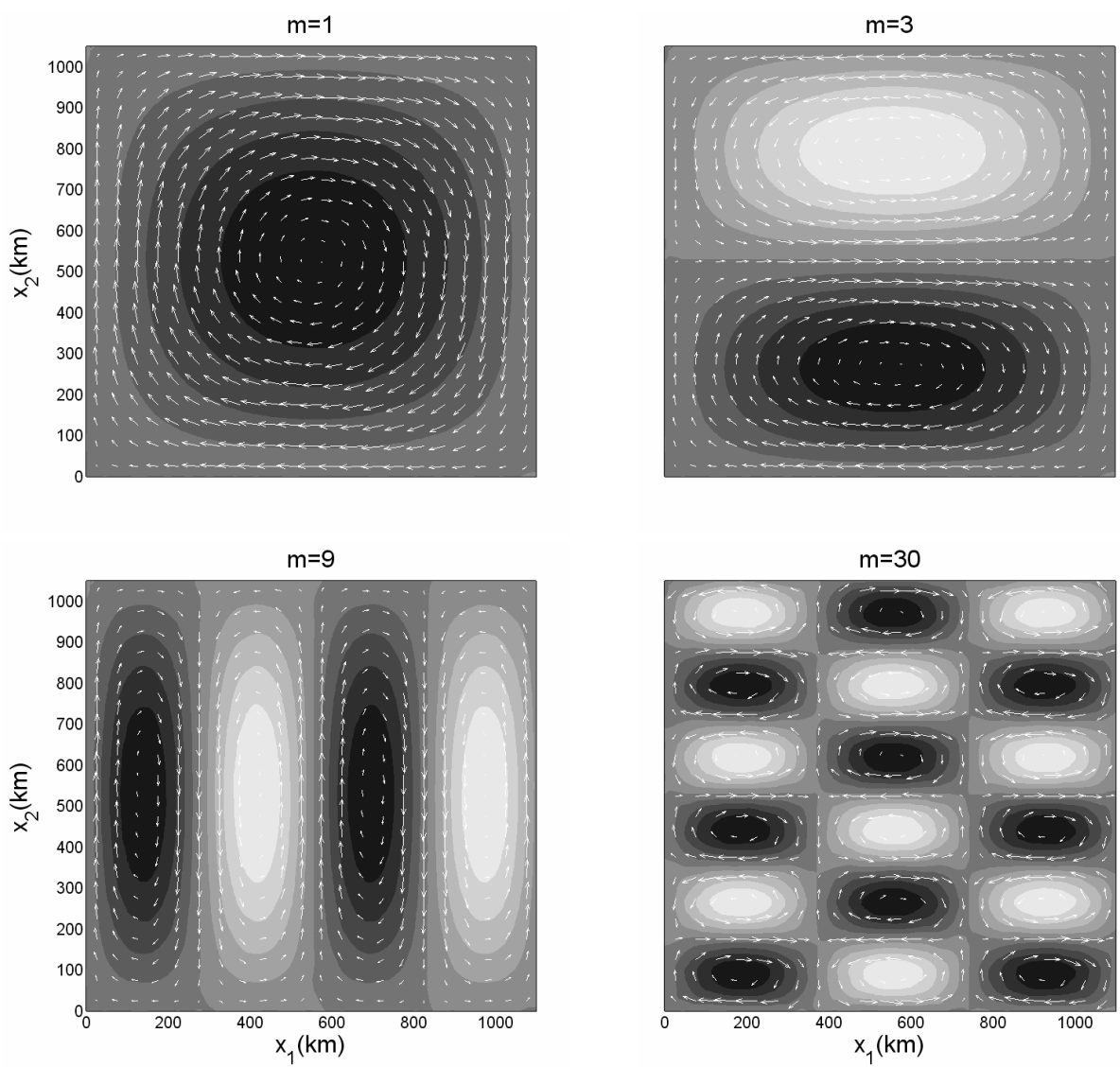

Fig. 5. Four orthonormal modes $\psi_{m}$ with numbers $m=1,3,9$, and 30 . The contour interval is non-dimensional, with positive vorticity in dark and negative vorticity in light, and non-dimensional velocity vectors are overlaid in each panel.

function (19) decays slower than the Gaussian one. This indicates that rare high-energetic wind events can strongly contribute to $w$ statistics. Therefore, even for small-amplitude errors, $\tau$-PDF has asymmetric shape with a tail stretching into short prediction time scales.

A weak second-order algorithm (Cao and Pope, 2003) is applied to numerical integration of stochastically forced Eqs. (6-8). Although the time step for the model integration is $5 \mathrm{~min}$, the stochastic wind is updated every hour. A correlation time $\left(t_{c}\right)$ for such a noise is shorter than one hour. Since characteristic time scales for the reference solution and PE are $t_{\text {ref }} \approx 10-20$ days (determined from Fig. 4a) and $t_{\text {error }} \approx 3-5$ days (determined from Fig. 7b), respectively, $t_{c} / t_{\mathrm{ref}} \ll 1$ and $t_{c} / t_{\mathrm{error}} \ll 1$. Such a stochastic wind represents a white noise-like process (Stratonovich, 1963).

PE statistics is insensitive to more often update of the stochastic wind. We made computations with update varying from less than one hour to $5 \mathrm{~min}$. These computations required very large computer resources. Therefore, the choice of one-hour update is a trade-off between accuracy in representation of the wind forcing and the computational cost.

Ensembles of perturbed model trajectories were used to compute $\tau$-PDF. Little difference in $\tau$-statistics is obtained between ensembles of $1 \times 10^{3}, 5 \times 10^{3}, 1 \times 10^{4}, 2 \times 10^{4}$, and $5 \times 10^{4}$ samples. The optimal size of an ensemble sampling, i.e. a number of ensemble realizations providing a tradeoff between the ensemble ability to reproduce main features of PE statistics, and the computational cost, is estimated as $10^{3}$ for any values of $\bar{\sigma}^{2}$. Hereafter the non-dimension variance of wind perturbations introduced as $\bar{\sigma}^{2}=\sigma^{2} / \sigma_{0}^{2}$, $\sigma_{0}^{2}=1.0 \mathrm{~m}^{2} \mathrm{~s}^{-2}$, is used. The optimal size is found using the non-symmetrical Kullback- Leibler distance (White, 1994). An interested reader is referred to Chu and Ivanov (2005), Ivanov and Chu (2007) for more details of such an approach.

\section{Model phase space}

For the chosen model parameters, a quasi-geostrophic approximation (Pedlosky, 1987) is applicable to interpret the reference and perturbed flows in a model phase space. The basis of M-dimension phase space is formed from orthonormal functions (modes) $\psi_{m}$, which are the eigenvectors of the plane Laplace operator $\nabla^{2}$ (Eremeev et al., 1991, 1992),

$$
\nabla^{2} \psi_{m}=-\lambda_{m} \psi_{m},\left.\quad \psi_{m}\right|_{\Gamma \cup \Gamma^{\prime}}=0, \quad m=1, \ldots, M .
$$


Then, the geostrophic stream function is decomposed as

$\psi\left(x_{1}, x_{2}, t\right)=\sum_{m=1}^{M} A_{m}(t) \psi_{m}\left(x_{1}, x_{2}\right)+\psi_{\text {harm }}\left(x_{1}, x_{2}, t\right)+C(t)$,

$M=100$,

where $\psi_{\text {harm }}$ is the harmonic function; the constant $C(t)$ is determined from the mass conservation constraint imposed upon the stream function (McWilliams, 1977).

The spatial modes $\psi_{m}$ are determined only by the geometry of a basin, and can be easily computed for any nonrectangular domain. Figure 5 shows the spatial structure of a few basis functions $\psi_{m}$ involved in the present analysis. The spatial modes, in general, have no physical significance by themselves, but only when they imply a flow. However, they are useful to identify the energy-dominated scales for the reference and perturbed flows.

The harmonic function $\psi_{\text {harm }}$ is obtained from

$$
\begin{aligned}
& \nabla^{2} \psi_{\text {harm }}=0 \quad,\left.\quad \psi_{\text {harm }}\right|_{\Gamma}=0, \\
& \left.\psi_{\text {harm }}\right|_{\Gamma^{\prime}}=-\int_{0}^{x_{2}} \bar{u}_{b}(t, y) d y,
\end{aligned}
$$

It is a highly predictable component of the flow because of the exact value of $\bar{u}_{b}$ in Eq. (22).

The PE presents the sum of the mean or systematic error $\psi_{\text {ref }}-\left\langle\psi_{\text {pert }}\right\rangle$ and the transient or random error $\delta \psi$ :

$$
\begin{aligned}
\left\langle I_{\text {pert }}^{2}\right\rangle & =\left\langle\left\|\psi_{\text {ref }}-\left\langle\psi_{\text {pert }}\right\rangle-\delta \psi\right\|^{2}\right\rangle \\
& =\left\|\psi_{\text {ref }}-\left\langle\psi_{\text {pert }}\right\rangle\right\|^{2}+\left\langle\|\delta \psi\|^{2}\right\rangle .
\end{aligned}
$$

The inertial (nonlinear) terms of the governing equations hardly contribute to $\left\langle\psi_{\text {pert }}\right\rangle$ at the initial stage of PE growth, and their contribution is negligible at later stages. The random error grows faster than the systematic error. Therefore, we suggest quantifying the PE behavior through the growth of the random error only.

The reference and perturbed solutions are represented in the model phase space as the reference $\boldsymbol{A}=\left[A_{1}(t), \ldots, A_{M}(t)\right]$ and error $\boldsymbol{a}=\left[a_{1}(t), \ldots, a_{M}(t)\right]$ trajectories, respectively. Using these notations the variance of the random prediction error becomes

$$
\langle\|\delta \psi\|\rangle=\sum_{m=1}^{M}\left\langle a_{m}^{2}\right\rangle,
$$

and the wind error source term (see Appendix A) is written by

$$
R_{m}=\gamma_{m}^{2}\left(b_{m}^{2}+c_{m}^{2}\right)
$$

where $\quad \gamma_{m}=\frac{\rho_{\text {air }}}{\rho_{w}} C_{d} H^{-1} \lambda_{m}^{-1} \sigma^{2}, \quad b_{m}=\iint \frac{\partial G}{\partial x_{1}} \psi_{m} d x_{1} d x_{2}$, $c_{m}=\iint \frac{\partial G}{\partial x_{2}} \psi_{m} d x_{1} d x_{2}$, the double integration is made over the semi-closed basin area.



Fig. 6. Phase portrait in a phase sub-space generated by the basis functions $\psi_{p}, \psi_{q}$ and $\psi_{s}$.

Applying the classical linear stability analysis (Guckenheimer and Holmes, 1983) to the model (6-8) linearized near the spin up solution, we find that the spin up represents an unstable focus (spiral point) in the model phase space. The growth and decay of infinitesimal perturbations near this point are characterized by the spectra of positive and negative local characteristic exponents only.

Therefore, an error trajectory should tend to this focus (denoted by B in Fig. 6) along stable manifolds corresponding to the large-scale negative exponents and simultaneously drifts from $B$ along the small-scale unstable manifolds corresponding to the positive exponents. Such a model trajectory is asymptotically unstable in Lyapunov sense as $t \rightarrow \infty$ (Guckenheimer and Holmes, 1983).

Figure 6 shows projections of error trajectories onto the phase subspace. The trajectories tend to reach the focus along the stable manifolds projected onto the phase plane $\left[a_{p}, a_{q}\right]$. However, they move away from the focus along unstable manifolds projected on the phase planes $\left[a_{p}, a_{s}\right]$ and $\left[a_{q}, a_{s}\right]$.

We use $M=100$ and confirm that such a choice does not smooth the reference trajectory for 70 days of model integration. The relative variance $S_{m}=\left(A_{p} \cdot A_{p}\right)_{p=1}^{m} \cdot I_{\text {ref }}^{-2}$ converges to 1 very quickly as $m$ increases (Fig. $4 \mathrm{~b}$ ). The first fifty-sixty modes contain more than $99 \%$ of variance for the reference solution.

One hundred mode representation is also quite sufficient to approximate the error trajectory for 6070 days of model integration. The relative variance $S_{m}^{\prime}=\left(<a_{p} \cdot a_{p}>\right)_{p=1}^{m} \cdot\left\langle I_{\text {pert }}^{2}\right\rangle^{-1}$ converges to 1 as $m$ increases, slower than $S_{m}$ (compare Figs. $4 \mathrm{a}$ and $7 \mathrm{a}$ ), but the speed of the convergence is quite high. 

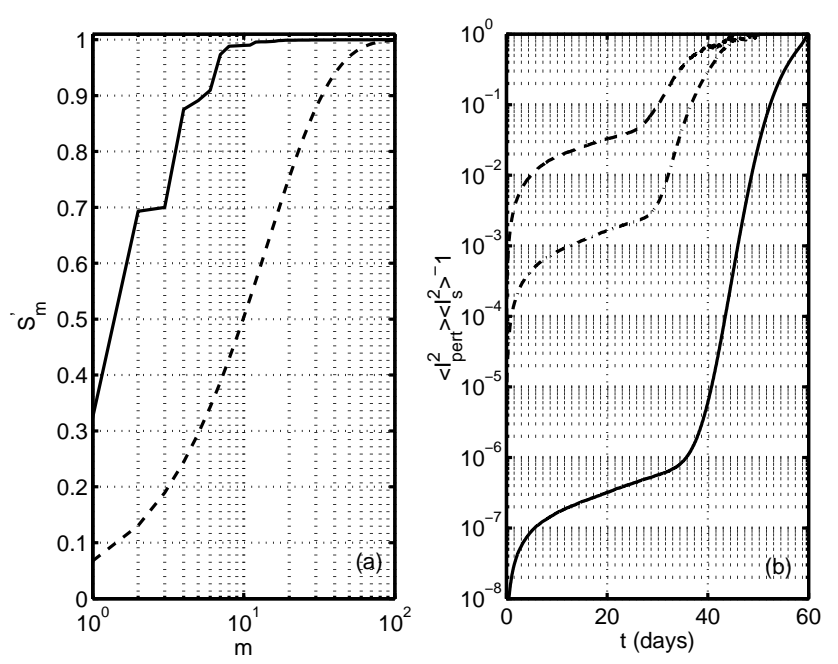

Fig. 7. Features of a perturbed solution: (a) the relative variance $S_{m}^{\prime}$ computed at day-10 (dashed curve) and day-50 (solid curve), $\bar{\sigma}^{2}=1.0$; (b) $<I_{\text {pert }}^{2}>$ normalized by its saturation value $<I_{s}^{2}>$ for $\bar{\sigma}^{2}=0.1$ (solid curve), 1.0 (dotted and dashed curve), and 2.0 (dashed curve).

\section{Error evolution}

Typical growth of an ensemble averaged PE with time for $G=G_{1}$ and $\bar{\sigma}^{2}$ varying between 0.1 and 2.0 , is given in Fig. 7b. Perturbations excited by uncertainty of stochastic wind grow at all scales and during the whole 50-60 day period. That is in contrast to the case when there is uncertainty in the initial condition only. In the last case the high predictability of the dynamical regime within the initial 15-20 day period was clearly demonstrated by Ivanov and Chu (2007): the PE at first decays with time for all scales due to dissipation caused by nonlinear bottom friction, and only after day-20 grows faster than [quasi]-exponentially. Therefore, the presence of the spatio-temporal noise (Eq. 10) in wind forcing (Eq. 9) causes the monotonic error growth shown in Fig. $7 b$.

At least four predictability regimes are identified from Fig. 7b. In all the cases the PE grows in a monotonic manner but with different speeds. More accurately, these regimes can be identified using the growth rate defined as $Q=\frac{d}{d t} \ln <I_{\text {pert }}^{2}>$.

A set of growth rates computed for different $G$ and $\bar{\sigma}^{2}$ is presented in Figs. 8a, b, c, d. These results clearly indicate that error dynamics strongly depends on the intensity and spatial inhomogeneity of wind uncertainty.

\subsection{Linear growth of perturbations}

At the initial stage (transient phase) where the stochastic forcing term dominates the governing equations, $Q \sim 1 / t$

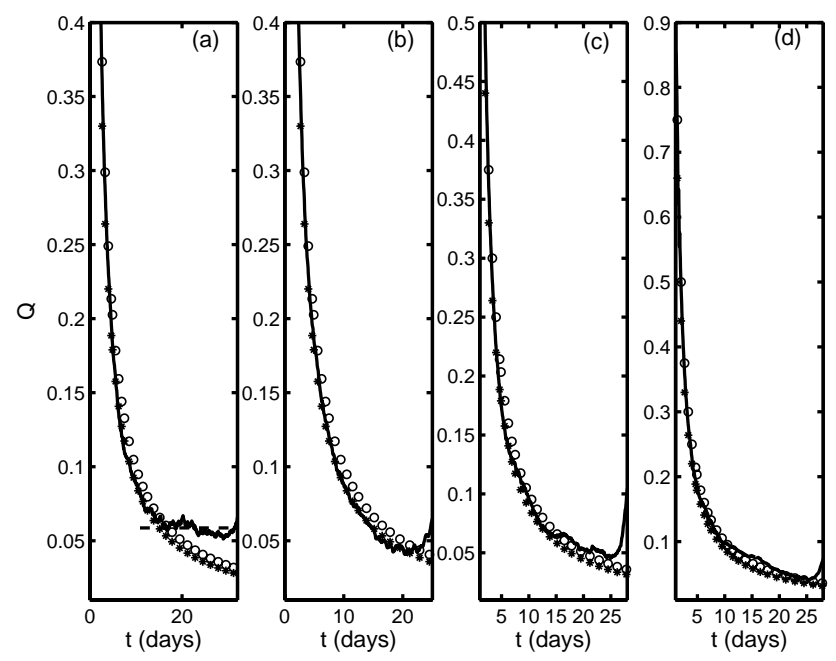

Fig. 8. The growth rate $Q$ (solid curve) for different $\bar{\sigma}^{2}$ and $G$ : (a) $\bar{\sigma}^{2}=0.1, G=G_{2}$; (b) $\bar{\sigma}^{2}=1.0, G=G_{1}$; (c) $\bar{\sigma}^{2}=1.0, G=G_{2}$, and (d) $\bar{\sigma}^{2}=2.0, G=G_{2}$. Dashed line, white dots and asterisks show exponential, linear, and power (with scaling exponent of $8.8 \times 10^{-1}$ ) laws, respectively.

(Figs. 8a, b, c, d). It corresponds to the linear growth of the mean square error:

$\left\langle I_{\text {pert }}^{2}\right\rangle \approx D_{\text {eff }} t$

Duration of this regime is typically up to $4-5$ days if $\bar{\sigma}^{2} \sim 0.1-$ 1.0. The effective coefficient $D_{\text {eff }}$ is determined by summation of contributions from the error source term at all wavenumbers $D_{\text {eff }}=\sum_{m=1}^{M} R_{m}$.

Linear law Eq. (26) was earlier documented in a number of studies (see, for example Vannitsem and Toth, 2002). We have analytically determined the wind error sources for model (6-8) (Appendix A). Our calculations show strong dependence of the effective coefficient on the variance of wind, as $\sim \sigma^{4}$, as well as on degree of spatial inhomogeneity of the wind forcing.

\subsection{Power growth of perturbations}

For moderate but inhomogeneous winds the power growth of perturbations are observed in our numerical experiments after the transient phase (for example, see Figs. 8a, b, c). For small values of $\bar{\sigma}^{2} \ll 1.0$ the power growth is replaced by the exponential growth (shown by the dashed line in Fig. 8a). If $\bar{\sigma}^{2}$ exceeds 1 , there is no exponential growth and the PE grows with the power law with power exponent of about $8.8 \times 10^{-1}$. This regime exists between day- 5 and day- 15 in Fig. 8a, between day-7 and day-23 in Fig. 8b, between day4 and day-14 in Fig. 8c, but there is no power-law regime in Fig. 8d when the stochastic wind uncertainty is too large 

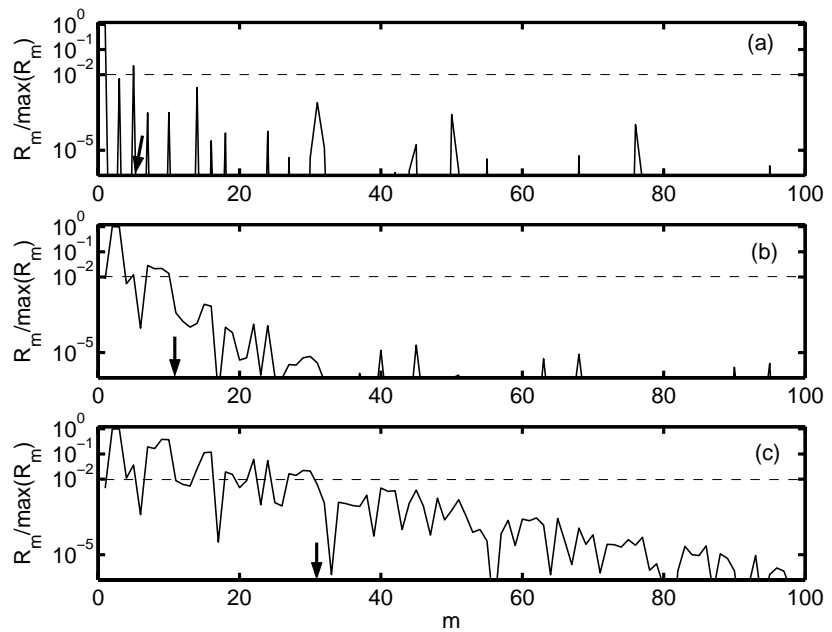

Fig. 9. Spectra of wind error term with (a) $G_{1}$, (b) $G_{2}$ and $\lambda=600 \mathrm{~km}$, and (c) $G_{2}$ and $\lambda=100 \mathrm{~km}$. A critical wavenumber is determined from the condition of $R_{m} / \max \left(R_{m}\right)=1.0 \times 10^{-2}$ (shown by dashed line). Black arrows indicate the critical numbers.

$\left(\bar{\sigma}^{2}>2.0\right)$. For such a variance the linear growth of PE dominates.

For $G=G_{1}$ the spectrum of $R_{m}$ is linear with dominating peaks at wavenumbers $m=1,3$, and 5 (Fig. 9a). These wavenumbers indicate modes with maximum response to the stochastic wind forcing. The weak wind forcing $\left(\bar{\sigma}^{2} \ll 1.0\right)$ essentially affects the large scales of the flow and excites only several low-order modes. In this case the PE at first grows linearly, then its quasi-exponential growth is observed. Smaller scales affected by the stochastic wind are subject to strong viscous damping due to increasing drag coefficient $\alpha$ with growth of the kinetic energy of large-scale perturbations. Therefore, the smaller scales grow slower than the unstable large scales. The growing perturbations rapidly adopt the horizontal scales comparable to those of the reference state.

Alternatively, stronger stochastic wind $\left(\bar{\sigma}^{2} \geq 1.0\right)$ excites more modes at smaller scales than the weak wind, and the coherent behavior of modes is clearly observed in this case (for example see Fig. 8b).

For $G=G_{2}$ and $\beta_{c}=600 \mathrm{~km}$, what corresponds to inhomogeneous winds, the spectrum of $R_{m}$ is continuous and band limited at the critical wavenumber $m=11$ (Fig. 9b). Therefore, the stochastic wind excites several modes around wavenumbers of 2 and 10. The PE growth ratio depends on the spectrum of local characteristic exponents. In this case the coherent behavior of modes exists even for the weak wind forcing $\left(\bar{\sigma}^{2} \ll 1.0\right)$.

The decorrelation scale $\beta_{c}$ determines the width of spectrum band for $R_{m}$. For example, the critical wavenumber equals to 32 if $\beta_{c}=100 \mathrm{~km}$ (Fig. 9c). Reduced values of $\beta_{c}$ lead to a wider spectrum of local characteristic exponents and stronger contribution of the cumulative effects to the PE growth.
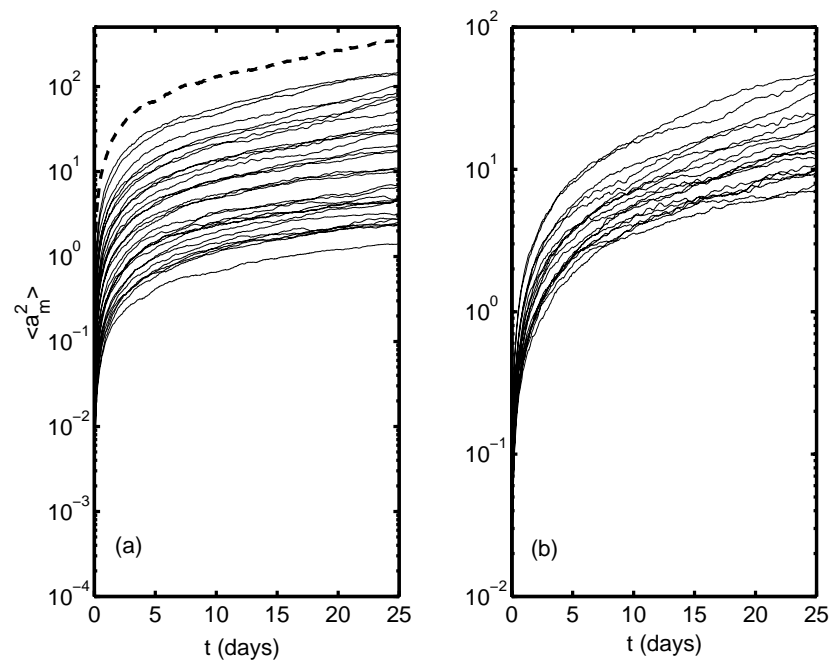

Fig. 10. Coherent behavior of perturbations on different scales for $\bar{\sigma}^{2}=1.0$ and $G=G_{2}$. (a) Large, and (b) small scale perturbations. The largest scale perturbation with $m=1$ is labeled by dashed curve.

Our computations have also shown that one of the main specificities of the power growth regime is that all dominating scales (modes) may exhibit a similar growth rate. For example, Fig. 10a, b demonstrates two groups of modes with different coherent behavior from day-7 to day- 25 . The coherent behavior of modes is a collective response to the external stochastic forcing when amplitudes of many modes exceed some threshold at the same time due to spatial inhomogeneity of wind forcing (defined by the choice of $G$ ). Clearly that the coherent behavior is absent if this threshold was exceeded only by a few modes.

\section{3 "Super-exponential" growth of perturbations}

After day-25 the PE grows faster than exponentially ("superexponentially") until non-linear interactions between different scales destroy this growth (saturation regime) (Fig. 7b). Strong coherence in behavior of different modes accompanies the "super-exponential" growth of perturbations.

Figure 11a shows coherent behavior of 30 dominated modes. Explicit "synchronization" in behavior of these modes is observed. After 35-37 days of integration, the first mode (shown by solid curve in Fig. 11a) is a "driver" determining the behavior of all other dominant large-scale modes, which are called "responses" (hereafter we use the terminology from Boccaletti et al., 2002). During a 10-12 day time period (up to day-47) the driver and responses perform coherently. For small-scale perturbations at least two drivers can be identified in Fig. 11b. Mode $m=9$ (the first driver) grows along the exponential law. The non-exponential growth of the second driver (mode $m=11$ ) is a consequence of the fact that different scales grow with different local characteristic exponents varying between $2.3 \times 10^{-1}$ day $^{-1}$ and $9.4 \times 10^{-1}$ day $^{-1}$. 

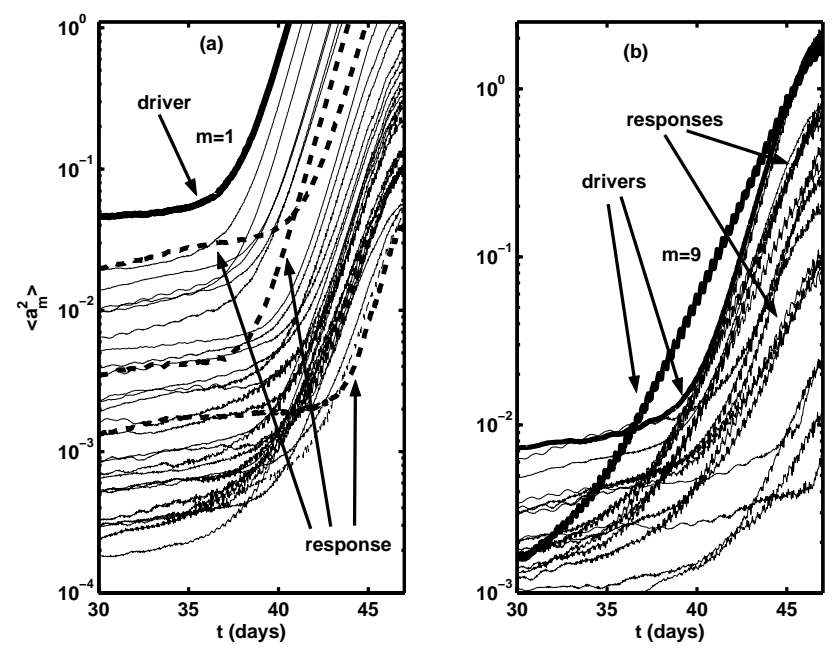

Fig. 11. Coherent behavior of perturbations on different scales for $\bar{\sigma}^{2}=0.1$ and $G=G_{2}$. (a) Mode $m=1$ is "the driver" for large-scale responses. Three responses (modes $m=4,12,29$ ) are indicated by bold dashed lines; (b) two drivers (modes $m=9,11$ ) observed for small-scale perturbations. Mode $m=9$ grows with the exponential law.

For very strong external noises the modes may not be synchronized because the intensive noise destroys correlations among them. This effect is clearly observed in our numerical experiments independently on $G$ if $\bar{\sigma}^{2}>2.0$.

\subsection{Physical mechanisms of perturbation growth}

The numerical results discussed above explicitly show "a synchronization" effect due to external noise and replacements of the traditional exponential growth of perturbations by the power or sub-exponential growth. There is a number of mechanisms for which noise can lead to more order in the dynamics. To be mentioned here are the effects of noise-induced order in chaotic dynamics (Matsumoto and Tsuda, 1983), synchronization of self-sustained oscillators (Pikovsky et al., 2000), cumulative effects of many different scales (Aurell et al., 1996), coherence resonance (Pikovsky and Kurths, 1997), stochastic resonance (Nicolis and Nicolis, 1981; Benzi et al., 1981), and interference between initial error and stochastic forcing (Seki et al., 1993). These effects in some respects are close and cannot be easily distinguished from one another when signals reflect different variables of the same system (Rosenblum et al., 2004).

In our case circulation dynamics is not driven by a periodical force. That allows excluding the stochastic resonance as a possible physical mechanism driving mode dynamics. The stochastic resonance appears if both periodic and noisy forces drive a nonlinear system, with the periodic response having a maximum at some noise amplitude.

Perez-Munuzuri et al. (2005) have demonstrated a coherent resonant behavior for an atmospheric global circulation model induced by a white (in time and space) additive Gaussian noise. In our case, however, no peak appears in the spectral density $\left\langle a_{m}^{2}\right\rangle$ at a given wavenumber $m$ for an intermediate level of noise.

Our results show the coherent behavior of a group of modes when amplitudes of many modes in the group exceed a threshold. Low- and high-order modes are separately grouped. For quite large amplitudes our results demonstrate the driver-response relationship for which phase of modes are locked.

To examine this mechanism we have linearized governing Eqs. (6-8), and calculate the growth of perturbations excited by stochastic forcing Eq. (10) in this case. The results of these calculations are summarized as follows. The power growth of perturbations in the transient regime is observed up to day-20 and, therefore, cannot be caused by the phase locking mechanism. "Super-exponential" growth of perturbations after day-25 disappears when nonlinear (inertial) terms are removed from the governing equations.

The power growth of perturbations in the transient regime can be explained using results obtained by Seki et al. (1993). They pointed out that for a linear dynamical system forced by a Gaussian white noise the mean deviation of perturbation amplitudes can grow along a power law up to a time scale $T_{b}$ defined by inverse of friction coefficient. For large values of friction coefficients such a power-law behavior disappears because $T_{b} \rightarrow 0$.

The effective dissipation in model (6-8) depends on the structure of the reference flow and shape of the spectrum of $R_{m}$. When forcing becomes stronger or is inhomogeneous, the center mass of energy spectrum shifts to high wavenumber domain and, in general, the dissipation of perturbations reduces because the nonlinear bottom friction is most effective for largest-scale perturbations as it has been checked numerically. That results into appearance of power-law behavior for variance of perturbations. In this case power exponent depends on the structure of time-dependent reference flow, and unfortunately, cannot be analytically calculated. Seki et al. (1993) calculated the power exponents for two simple stochastic dynamical systems only. However explicit correlation between level of model dissipation and existence of a power-law behavior of perturbations are clearly observed in our numerical experiments.

After 25 day integration noiseless model (6-8) reaches a spin up when a solution oscillated with a period of 120 days for the dominant low-order modes within any 1000-day time interval. Oscillating modes weakly interact one with another due to nonlinear (inertial and frictional) terms in the governing equations. Effects of external noise on these modes lead to coherent behavior of modes that seems to be similar to the phase locking of the modes, which is understood in a statistical sense, as the existence of a preferred value of the phase difference between individual stochastically forced modes with weak interactions among them. 
Non-steady nature of the reference flow is essential for existence of super-exponential regime of perturbation growth. This was checked by numerical modeling. The oscillations are smoothed and the reference flow becomes steady when the drag coefficient is 2-3 times as much. Neither "super exponential" growth of perturbations nor coherent behavior of modes is observed for such levels of model dissipation.

Note that high-order modes of the reference flow have a dominant oscillation period of about 30 days, not 120 days as low-order modes. Since the first twenty modes contain up to $90 \%$ of the kinetic energy of circulation, 120-day oscillations dominate the flow and mask faster motions. However, existence of the second dominant period leads to coherence in behavior of high-order modes, which is different than that low-order modes demonstrate.

The coherence in behavior of modes can be explained by a number of mechanisms, such as synchronization of weakly coupled oscillators (Pikovsky et al., 2000), modulation (Landa, 1996) and others. In practice these mechanisms cannot be selected using only a driver-response relationship or the cross-spectral technique without a simple physical model. Unfortunately, this is a great problem to develop a model, which would adequately describe nonlinear oceanic flow dynamics with many degrees of freedom. Therefore herein we are not able to select one of the physical mechanisms discussed in modern literature for explanation of the coherent behavior of modes observed in our numerical experiments for finite-amplitude perturbations. However, undoubtedly the observed coherent behavior is due to nonlinear interactions among modes and oscillations of the reference flow.

\section{Finite-amplitude-induced transition in predictability skill}

Our computations have shown that for strong winds model predictability demonstrates stronger sensitivity to amplitudes of perturbations induced by the stochastic wind than to the choice of spatial structure function in Eq. (10). Intuitively, larger-amplitude perturbations should cause faster decay of model predictability, what was confirmed by our numerical experiments for the mean predictability time. $\tau$-mean monotonically reduced as $\bar{\sigma}^{2}$ increases (not shown).

Furthermore, our experiments have also shown that the collective behavior of finite-amplitude perturbations may cause sudden changes (bifurcations) in the high-order statistics of predictability time. This effect is clearly observed when perturbation amplitudes exceeded some threshold, after which the global correlations among the perturbations with different scales dominated the PE characteristics.

We have called such bifurcations as the "finite-amplitude induced phase (non thermodynamic) transitions in model predictability". They are detected using statistics of IPT,
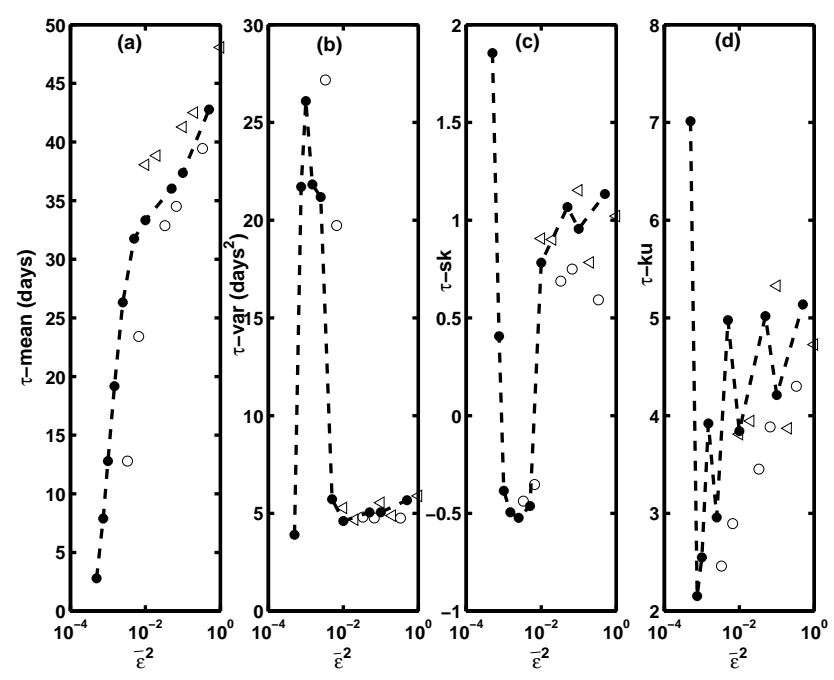

Fig. 12. $\tau$-statistics for different values of $\bar{\varepsilon}^{2}$ and $\bar{\sigma}^{2}$. (a) $\tau$-mean, (b) $\tau$-variance, (c) $\tau$-skewness and (d) $\tau$-kurtosis. Triangles, asterisks and circles correspond to $\bar{\sigma}^{2}=2.0,1.0$, and 0.5 .

such as $\tau$-variance, $\tau$-skewness and $\tau$-kurtosis, but other measures of model predictability are also applicable.

Finite-amplitude phase transitions should easily be detected for any hydrodynamic model using the dependence of IPT statistics on $\bar{\varepsilon}^{2}$ because the value of the tolerance level limits the maximum amplitude of perturbations existing in the model. As an example, detection of the phase transition for model (6-8) is described below.

For small tolerance $\left(\bar{\varepsilon}^{2}<5.0 \times 10^{-3}\right)$ model predictability is low: $\tau$-mean does not exceed 20 days (Fig. 12a) and $\tau$ variance is quite large, up to $25-27$ days $^{2}$ (Fig. 12b). Additionally, a large negative skewness about -0.5 (Fig. 12c) indicates that the IPT distribution has a tail stretching into domain of small prediction times.

The mean IPT monotonically grows as $\bar{\varepsilon}^{2}$ increases (Fig. 12a). Although here, the IPT grows with various rates for different tolerance levels, no bifurcations are observed in this figure. In contrast to $\tau$-mean, the value of $\tau$-variance suddenly changes when $\bar{\varepsilon}^{2}$ becomes larger than $5.0 \times 10^{-3}$ (this value is taken as a threshold). The variance, which was quite large (about 25-27 day ${ }^{2}$ ) for small tolerances, suddenly reduces to 5 day $^{2}$ when $\bar{\varepsilon}^{2}$ crosses the threshold (Fig. 12b).

Both $\tau$-skewness and $\tau$-kurtosis also change considerably (Figs. 12c, d). They converge asymptotically to 1.0 and 4.7 , respectively, as $\bar{\varepsilon}^{2}$ increases. Positive skewness corresponds to asymmetric $\tau$-PDF shapes with a tail stretching into large prediction times. The large kurtosis (much larger than 3 ) indicate that PDF is highly non-Gaussian.

Typical $\tau$-PDFs computed before and after the phase transition, are given in Figs. 13a and b, respectively. Comparing them one to another, we find that the phase transition causes the PDF tail stretching into domain of large prediction times. This tail is formed by rare predictions of duration up to 50 

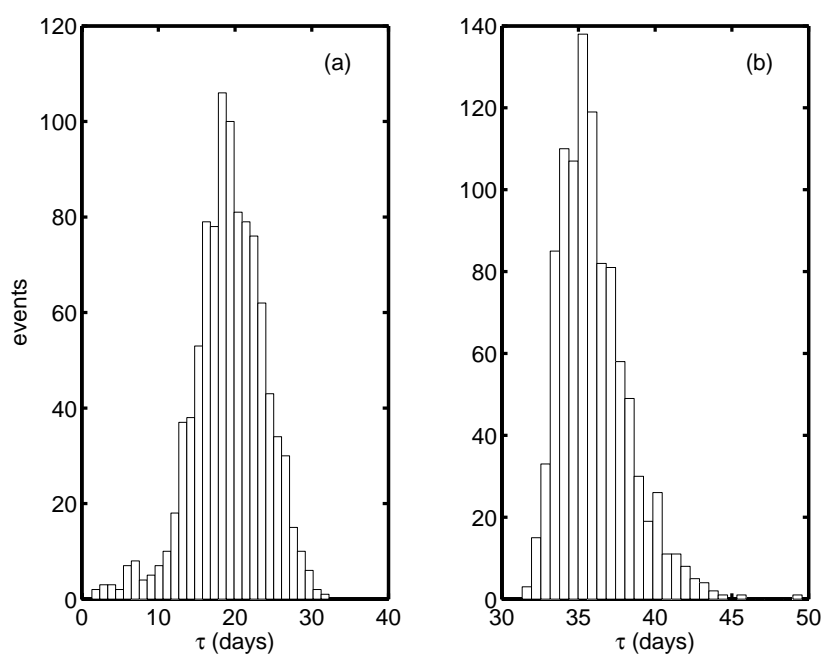

Fig. 13. Histograms of IPT computed for $\bar{\sigma}^{2}=1.0$. (a) $\bar{\varepsilon}^{2}=1.0 \times 10^{-2}$, (b) $\bar{\varepsilon}^{2}=0.1$.

days. Smaller variance and larger positive skewness show that model predictability was considerably enhanced after the phase transition.

Two physical mechanisms are responsible for the phase transition. First, it is clearly from Fig. 12a that the phase transition exists when the mean $\tau$-IPT is not less than 30 days. During this time period the model reaches the spin up state. Different stability properties in the phase space near and far away from the point are caused by the inhomogeneity of model phase space. That leads to different statistics of PE before and after the phase transition. Second, even when perturbation amplitudes are several percents of the reference solution, a coherent behavior of different scales are clearly observed. In this case $\tau$-variance reduces due to strong correlations among the scales (Kravtsov, 1993).

\section{Weibullian statistics of IPT}

Our computations have shown that stochastic forcing Eq. (10), in general, induces highly non-Gaussian $\tau$-PDFs for finite-amplitude PEs. The following question arises: what kind of statistics can be used to represent such $\tau$-PDFs? If appropriate distribution function is found, it would be possible to identify the ensemble generated PDFs from limited observation series and small forecast ensembles, and in turn to estimate the model predictability horizon (i.e. maximum predictability time reached for the given model and wind uncertainty (Kravtsov, 1993)).

We apply the three-parameter Weibull statistics with distribution $f(\tau)$ and cumulative distribution $P(t)$

$f(\tau)=\frac{\beta}{\eta}\left(\frac{\tau-\gamma}{\eta}\right)^{\beta-1} \exp \left[-\left(\frac{\tau-\gamma}{\eta}\right)^{\beta}\right]$,
$P(t)=\exp \left[-\left(\frac{t-\gamma}{\eta}\right)^{\beta}\right]$,

for the analysis of $\tau$. Here, $\eta, \gamma$, and $\beta$ are scale, shape, and location parameters (von Storch and Zwiers, 1999). The following original algorithm is developed to estimate the parameters of distribution (27) from an IPT ensemble sampling.

Closeness of two inverses of $\tau$-CDFs: $X(P)$ ("real" value) and $X_{0}(P)$ (the first guess), may be estimated by the Kullback-Leibler distance $\Omega$ (White, 1994):

$\Omega=\int_{0}^{1} X(P) \ln \left[X(P) / X_{0}(P)\right] d P$.

Then, $X(P)$ is the solution of the following variation problem (Kapur and Kesavan, 1992)

$\Omega \rightarrow \min$.

The Kullback-Leibler distance $\Omega$ should be a subject to additional constraints from the following condition: the probability weighted moments computed from the ensemble sampling $\left(\alpha_{1}, \alpha_{2}\right.$, and $\left.\alpha_{3}\right)$ and theoretically $\left(\hat{\alpha}_{1}, \hat{\alpha}_{2}\right.$, and $\left.\hat{\alpha}_{3}\right)$ from Eq. (4) must coincide. This condition is accounted for in Eq. (29) through additional constrain as

$$
\begin{aligned}
\Omega= & \int_{0}^{1} X(P) \ln \left[X(P) / X_{0}(P)\right] d P+\chi_{1}\left(\alpha_{1}-\hat{\alpha}_{1}\right) \\
& +\chi_{2}\left(\alpha_{2}-\hat{\alpha}_{2}\right)+\chi_{3}\left(\alpha_{3}-\hat{\alpha}_{3}\right) \rightarrow \min .
\end{aligned}
$$

where $\chi_{1}, \chi_{2}$, and $\chi_{3}$ are Lagrange multipliers.

Functional Eq. (30) is minimized with respect to $X(P)$. The solution of minimization problem (Eq. 30) is written as

$X(P)=X_{0}(P) \exp \left(-\chi_{1} P-\chi_{2} P^{2}-\chi_{3} P^{3}\right)$,

For details see Kapur and Kesavan (1992). Then, the Lagrange multipliers $\chi_{1}, \chi_{2}$, and $\chi_{3}$ are determined by the quasi-Newton iteration method as a solution of nonlinear least-square problem resulting from Eqs. (30) and (31).

Our computations show that (a) the method discussed above is robust relative to sampling error if only few moments are used as constrains, and (b) Lagrange multipliers are estimated within 10-12 iterations only. In general case, when sampling error is considerable and more moments are required in Eq. (30), the non-linear least-squares minimization problem is solved through the Levenberg-Marquardt iterative method (Engl et al., 1996).

We did not find difference between the distribution function calculated by a non-parametrical technique based on the Epanichenikov's kernel and the bootstrap re-sampling procedure (Good, 2001) directly from ensemble sampling, and appropriate Weibull counterpart obtained by the method (2931 ), at least at $95 \%$ confidence level. 
This conclusion is illustrated for the ensemble sampling shown in Fig. 14a. The $\tau$-CDFs computed by the nonparametrical technique (solid curve) and our method (black dots) are compared in Fig. 14b. Differences between them are negligible. The parameters $\eta, \gamma$, and $\beta$ are equal to $37.1 \times 10^{-1}$ days, 30.0 days and $16.7 \times 10^{-1}$, respectively.

Parameter $\beta$ affects the length of the PDF tail formed by rare forecasts, which are longer than the mean ensemble forecast $\langle\tau\rangle$. Small $\beta$ indicates enhanced probability for realization of abnormal long (in our case up to 50 days) model forecasts.

\section{Model predictability horizon}

Asymptotic behavior of $\tau-\mathrm{CDF}$ as $t \rightarrow \infty$ determines the predictability horizon of the model, i.e. the maximum predictability time of an individual forecasting for the given model and statistics of wind perturbations (Kravtsov, 1993).

Accordingly to Eq. (28) the model predictability horizon is calculated by

$\tau_{\text {hor }}=\gamma+\eta\left[-\ln \bar{P}^{*}\right]^{1 / \beta}$,

where $\bar{P}^{*}$ is the probability that $\tau_{\text {hor }}$ will be achieved in an individual forecasting. For fixed $\bar{P}^{*}$ Eq. (32) shows a slow power-law growth of the predictability horizon with the decrease of the shape parameter.

Let us estimate the predictability horizon for the example discussed above. Substitution of the distribution parameters obtained above into Eq. (32) leads to

$\tau_{\text {hor }} \approx 40.2$ days, 45.4 days and 50.5 days

for $\bar{P}^{*}=1.0 \times 10^{-2}, 1.0 \times 10^{-3}$ and $1.0 \times 10^{-4}$, respectively. These estimations demonstrate that for the chosen values of $\bar{\varepsilon}^{2}$ and $\bar{\sigma}^{2}$, the model predictability horizon is limited to 50 days, and any individual forecasting, which is longer than 50 days, is unlikely.

\section{Conclusions}

A simple shallow-water model was used to understand sensitivity and predictability of ocean models with inaccurate wind forcing. This model used a highly idealized representation of ocean dynamics and did not simulate the redistribution of PE between barotropic and baroclinic dynamics as high-resolution ocean models. However, due to the small number of degrees of freedom of the model (only 462 variables), distribution functions for predictability scale and its high-order moments were computed for a large number of ensemble realizations (up to 50000). This guaranteed reduced sampling error and robustness in estimating the PE statistics.
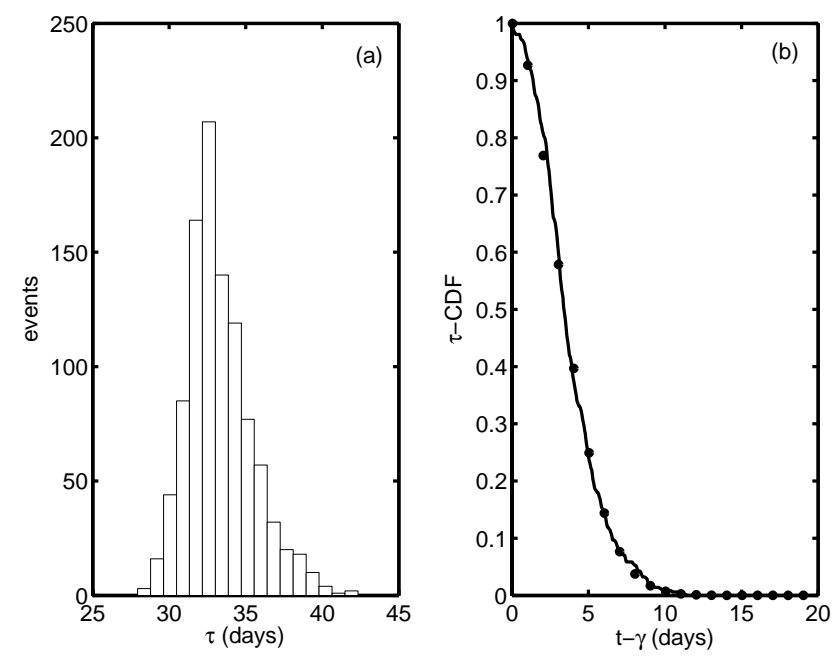

Fig. 14. Identification of $\tau$-CDF. (a) IPT histogram for a $10^{3}$ term ensemble; (b) CDF computed directly from the ensemble (solid curve) and using the developed method (black dots), $\gamma=30$ days.

Similar analysis is difficult to undertake in full-scale numerical forecast ocean models due to limited computer resources. Generally, the full-scale models produce small ensemble samples and therefore, cannot resolve the full complexity of the PE. The idealized model revealed trends in PE behavior and the model reconstructed PE statistics with minimum distortion. These statistics can be used for the analysis of the smaller ensemble samples from the full-scale models.

Baroclinic high-resolution ocean models should be used to examine the following trends obtained in the present study.

The predictability time for small perturbations may be much larger than the inverse of the leading local characteristic exponent. The shallow water model showed that it is possible to have non-trivial time evolutions of small (but finite) perturbations and that their growth could be fitted by power laws although the perturbations were actually amplified by the background flow. The power growth for all or a portion of scales was determined by the cumulative effects of multiple characteristic times .

The expected growth of error and decay of skill occurs most rapidly for smaller scales and, with time, expands to larger scales. One of the main features of the ocean is the existence of strongly interacting spatial scales, which raises the possibility of different behavior of the PE at different scales of motion. It is traditionally assumed that small scales are less predictable than larger scales. This picture was drawn by Lorenz (1969) for perfect model scenario, and then transferred to the analysis of forecast error as a function of spatial scale in operational atmospheric (see, for example Dalcher and Kalnay, 1987) and oceanographic models (Brasseur et al., 1996, among others).

Our simplified model predicts existence of two additional predictability regimes for imperfect models. For large scale 
stochastic winds, the perturbations rapidly grew to horizontal scales comparable to those of the reference state. In contrast, smaller scale perturbations excited by the wind were subject to strong viscous damping. Therefore, the predictability of wind-driven circulation was less affected by model uncertainties acting at small scales than at larger scales. Mahadevan et al. (2001), using a quasi-geostrophic ocean circulation model for perfect-model twin experiments, found that such a scenario was favourable for weakly aperiodic, periodic, and stationary circulation regimes when the mesoscale energy content was relatively low.

For stochastic wind that is limited to scales smaller than those occupied by large-scale flow, perturbations on different scales may grow coherently due to interactions among them. The coherent growth of perturbations has been identified on different scales at various stages of PE evolution. Coherent behavior of PE can be found in full-scale operational atmospheric models (Boer, 2003, as an example), and quasi-geostrophic models (Vannitsem and Nicolis, 1997; McWilliams and Chow, 1981, among others). McWilliams and Chow (1981) demonstrated that for a simple three-level quasi-geostrophic model, all scales of motion exhibited a similar growth rate after a short transient phase. The coherent growth of PE on different scales can be found in imperfect quasi-geostrophic models too (for example, see Vannitsem, 2006). These and other results (not discussed here) indicate that the coherent growth of PE may be caused by model independent (universal) mechanisms, which require further investigation.

The present study introduced a new statistics (Weibull) for finite-amplitude PE and suggested a practical way for its identification through the probability weighted moments and a variation principle. This extremum statistics is often observed to arise in finite sized, multi-body systems, exhibiting correlation over a broad range of scales, leading to emergent phenomenology, such as self-similarity and in some cases fractional dimensions (Boffetta et al., 2002). A universal approach to extract extremum statistics from short- and intermediate marine forecasts was suggested. Possible generalization of the approach for small forecast ensembles will be discussed in a separate paper.

\section{Appendix A}

\section{Wind error source term}

Using the quasi-geostrophic approximation, governing Eqs. (6-8) can be re-written in the spectral form

$\frac{d a_{m}}{d t}=Z_{m}\left(a_{k}\right)+Q_{m}\left(a_{l}, a_{k}\right)+r_{m}(t)$,

where $Z_{m}$ and $Q_{m}$ are linear and nonlinear (inertial and frictional) terms, respectively (for details see Pedlosky, 1987), and

$r_{m}=\gamma_{m} z\left(b_{m} \sin \theta-c_{m} \cos \theta\right)$,

$\gamma_{m}=\frac{\rho_{\mathrm{air}}}{\rho_{w}} C_{d} H^{-1} \lambda_{m}^{-1} \sigma^{2}, \quad b_{m}=\iint \frac{\partial G}{\partial x_{1}} \psi_{m} d x_{1} d x_{2}$,

and

$c_{m}=\iint \frac{\partial G}{\partial x_{2}} \psi_{m} d x_{1} d x_{2}$.

The stochastic processes $z(t)$ and $\theta(t)$ are defined in Sect. 4 . The variance of $\mathrm{PE}<a_{m}^{2}>$ satisfies the obvious equation

$\frac{d<a_{m}^{2}>}{d t}=2\left(<Z_{m} a_{m}>+<Q_{m} a_{m}>+<r_{m} a_{m}>\right)$,

with the wind error source term

$R_{m}=\gamma_{m}\left[b_{m}\left\langle z \sin \theta a_{m}\right\rangle+c_{m}\left\langle z \cos \theta a_{m}\right\rangle\right]$.

Since $f(z, \theta)=f(z) f(\theta)$, average of Eq. (A5) can be divided into two steps. (1) $r_{m} a_{m}$ is averaged over $z$. (2) The obtained function $<r_{m} a_{m}>_{z}$ is averaged over $\theta$.

The correlation function $\left\langle z a_{m}\right\rangle_{z}$ is analytically calculated using the cumulant decomposition (Klyatskin, 2005):

$$
\begin{aligned}
\left\langle z(t) a_{m}(t)\right\rangle_{w_{r}}= & \sum_{s=1}^{\infty}\left(\frac{1}{s !}\right) \int_{t_{o}}^{t} \ldots \int_{t_{o}}^{t} d t_{1} \ldots d t_{s} \kappa_{s+1}\left(t, t_{1}, \ldots, t_{s}\right) \\
& \left\langle\frac{\delta^{s} a_{m}(t)}{\delta z\left(t_{1}\right) \ldots \delta z\left(t_{s}\right)}\right\rangle_{z}
\end{aligned}
$$

Here, $\kappa_{s}\left(t_{1}, \ldots, t_{s}\right)$ is the s-th order cumulant of the noise $z$. The notation $\frac{\delta F}{\delta z}$ denoted the functional derivative.

For a stationary $\delta$-correlated noise $z$ one obtains

$\kappa_{s}\left(t_{1}, \ldots, t_{s}\right)=k_{s}\left(t_{1}\right) \delta\left(t_{1}-t_{2}\right) \ldots \delta\left(t_{s}-t_{s-1}\right)$,

where $k_{s}\left(t_{1}\right)$ are the intensity coefficients (Stratonovich, 1963).

Substituting Eq. (A7) into Eq. (A6) yields

$\left\langle z(t) \delta \mathrm{a}_{m}(t)\right\rangle_{z}=\sum_{s=1}^{\infty}\left(\frac{1}{s !}\right) k_{s+1}(t)\left\langle\frac{\delta^{s} a_{m}(t)}{\delta z(t)^{s}}\right\rangle_{z}$,

From Eq. (A1) we obtain for $s=1$

$\left\langle\frac{\delta a_{m}(t)}{\delta z(t)}\right\rangle_{z}=\gamma_{m}\left(b_{m} \cos \theta+c_{m} \sin \theta\right)$,

and for $s \geq 2$

$\left\langle\frac{\delta^{s} a_{m}(t)}{\delta z(t)^{s}}\right\rangle_{z}=0$.

Taking into consideration that for exponential distribution function the s-order cumulant is calculated as $\kappa_{s}=2 \cdot(s-1)$ ! (Zelen and Severo, 1972), and averaging Eq. (A9) over the stochastic process $\theta(t)$ we find

$R_{m}=\gamma_{m}^{2}\left(b_{m}^{2}+c_{m}^{2}\right)$, 
The coefficients $b_{m}$ and $c_{m}$ depend only on the spatial inhomogeneity of stochastic wind forcing. Spectrum of wind error term $R_{m}$ for different structure functions $G$ is shown in Figs. 9a, b, c.

Acknowledgements. L. Ivanov thanks the NSF for the support through Awards N OCE-0530748. This study was supported by the Office of Naval Research, Naval Oceanographic Office, and the Naval Postgraduate School for P. Chu. Comments of both reviewers were useful and helped to improve our presentation. We thank O. Talagrand for his critical comments on the paper draft.

Edited by: O. Talagrand

Reviewed by: two anonymous referees

\section{References}

Auclair, F., Marsaleix, P., and De Mey, P.: Space-time structure and dynamics of the forecast error in a coastal circulation model of the Gulf of Lions, Dyn. Atmos. Oceans, 36, 309-346, 2003.

Aurell, E., Boffetta, G., Crisanti, A., Paladin, G., and Vulpiani, A.: Predictability in systems with many characteristic times: The case of turbulence, Phys. Rev. E, 53, 2337-2349, 1996.

Benzi, R., Sutra, A., and Vulpiani, A.: The mechanism of stochastic resonance, J. Phys. A: Math. Gen., 14, L453-L457, 1981.

Berloff, P. S.: Random-forcing model of the mesoscale oceanic eddies, J. Fluid Mech., 529, 71-95, 2005.

Berloff, P. S. and McWilliams, J. C.: Large-scale, low-frequency in wind-driven ocean gyres, J. Phys. Oceanogr., 29, 1925-1949, 1999.

Blumberg, A. F. and Mellor, G. L.: A description of a threedimensional coastal ocean circulation model, in Three Dimensional Coastal Ocean Models, American Geophysical Union, Washington D.C., 1-16, 1987.

Boccaletti, D., Kurths, J., Osipov, G., Valladares, D. L., and Zhou, C. S.: The synchronization of chaotic systems, Phys. Rep., 366, 1-101, 2002.

Boer, G. J.: Predictability as a function of scale, Atmos. Ocean, 41, 203-215, 2003.

Boffetta, G., Celani, A., Cencini, M., Lacorata, G., and Vulpiani, A.: The predictability problem in systems with an uncertainty in the evolution law, J. Phys. A: Math. Gen., 33, 1313-1324, 2000.

Boffetta, G., Cencini, M., Falconi, M., and Vulpiani, A.: Predictability: A way to characterize complexity, Phys. Rep., 356, 367-474, 2002.

Bogden, P. S.: The impact of model-error correlation on regional data assimilative models and their observational arrays, J. Mar. Res., 59, 831-857, 2001.

Brasseur, P., Blayo, E., and Verron, J.: Predictability experiments in the North Atlantic Ocean: Outcome of a quasi-geostrophic model with assimilation of TOPEX/POSSEIDON altimeter data, J. Geophys. Res., 101, 14 161-14 173, 1996.

Burillo, I. A., Caniaux , G., Gavart, M., De Mey, P., and Baraille, R.: Assessing ocean-model sensitivity to wind forcing uncertainties, Geophys. Res. Lett., 29, 5-1-5-4, 2002.

Cao, R. and Pope, S. B.: Numerical integration of stochastic differential equations: Weak second-order mid-point scheme for application in the composition PDF method, J. Comput. Phys., $185,194-212,2003$.
Capotondi, A. and Holland, W. R.: Decadal variability in an idealized ocean model and its sensitivity to surface boundary conditions, J. Phys. Oceanogr., 27, 1072-1093, 1997.

Chu, P. C. and Ivanov, L. M.: Statistical characteristics of irreversible predictability time in regional ocean models, Nonlin. Processes Geophys., 12, 129-138, 2005, http://www.nonlin-processes-geophys.net/12/129/2005/.

Chu, P. C., Fan, C. W., and Ehret, L. L.: Determination of open boundary conditions from interior observational data, J. Atmos. Ocean. Technol., 14, 723-734, 1997.

Chu, P. C., Chen, Y. C., and Liu, W. T.: Uncertainty of the South China Sea prediction using NSCAT and NCEP winds during Tropical Storm Ernie 1996, J. Geophys. Res., 104, 11273 $11289,1999$.

Chu, P. C., Ivanov, L. M., Margolina, T. M., and Melnichenko, O. V.: On probabilistic stability of an atmospheric model to various amplitude perturbations, J. Atmos. Sci., 59, 2860-2873, 2002.

Dalcher, A. and Kalnay, E.: Error growth and predictability in operational ECMWF forecasts, Tellus, 39A, 474-491, 1987.

Engl, H. W., Hanke, M., and Neubauer, A.: Regularization of Inverse Problems, Springer, New York, 321 pp., 1996.

Eremeev, V. N., Ivanov, L. M., and Melnichenko, O. V.: Modal decomposition for three-dimension fields in ocean, Dokl. Akad. Nauk SSSR+, 310, 1453-1456, 1991.

Eremeev, V. N., Ivanov, L. M., and Kirwan Jr., A. D.: Reconstruction of oceanic flow characteristics from quasi-Lagrangian data, I: Approach and mathematical methods, J. Geophys. Res., 97, 9733-9742, 1992.

Freidlin, M. I. and Wentzell, A. D.: Random Perturbations of Dynamical Systems, Springer-Verlag, 448 pp., 1998.

Gardiner, C. W.: Handbook of Stochastic Methods for Physics, Chemistry and the Natural Sciences, Springer-Verlag, Berlin; New York, 442 pp., 1985.

Good, P. I.: Re-sampling methods: a practical guide to data analysis, Birkhauser, Boston, 238 pp., 2001.

Greenwood, J. A., Landwehr, J. M., Matalas, N. C., and Wallis, J. R.: Probability-weighted moments: definition and relation to parameters of several distributions expressible in inverse form, Water Resour. Res., 15, 1049-1054, 1979.

Guckenheimer, J. and Holmes, Ph.: Nonlinear Oscillations, Dynamical Systems, and Bifurcations of Vector Fields, SpringerVerlag, New York, 459 pp., 1983.

Holland, W. R. and Malanotte-Rizzoli, P.: Along-track assimilation of altimeter data into an ocean circulation model: Space versus time resolution studies, J. Phys. Oceanogr., 19, 1507-1534, 1989.

Hosking, J. R. M. and Wallis, J. K.: Regional Frequency Analysis: An Approach Based on L-Moments, Cambridge Univ. Press, Cambridge, 240 pp., 1997.

Ivanov, L. M., Kirwan Jr., A. D., and Melnichenko, O. V.: Prediction of the stochastic behavior of nonlinear systems by deterministic models as a classical time-passage probabilistic problem, Nonlin. Processes Geophys., 1, 224-233, 1994, http://www.nonlin-processes-geophys.net/1/224/1994/.

Ivanov, L. M. and Chu, P. C.: On stochastic stability of regional ocean models with finite-amplitude perturbations of initial conditions, Dyn. Atmos. Oceans, 43(3-4), 199-225, 2007.

Jiang, S. and Malanotte-Rizzoli, P.: On the predictability of regional oceanic jet stream: The impact of model errors at the inflow 
boundary, J. Mar. Res., 57, 641-669, 1999.

Kapur, J. N. and Kesavan, H. K.: Entropy Optimization Principles with Applications, Academic Press, San Diego, 408 pp.,, 1992.

Klyatskin, V.: Dynamics of Stochastic Systems, Elsevier, Amsterdam, 212 pp., 2005.

Kravtsov, Yu. A.: Limits of Predictability, Springer-Verlag, BerlinNew York, 252 pp., 1993.

Landa, P. S.: Nonlinear Oscillations and Waves in Dynamical Systems, Kluwer Academic Publishers, Dordrecht-Boston-London, 564 pp., 1996.

Lorenz, E. N.: Irregularity. A fundamental property of the atmosphere, Tellus, 36A, 98-110, 1984.

Lorenz, E. N.: The predictability of a flow which possesses many scales of motion, Tellus, 21, 289-307, 1969.

Lorenz, E. N.: Deterministic non-periodic flows, J. Atmos. Sci., 20, 130-141, 1963.

Mahadevan, A., Lu, J., Meacham, S. P., and Malanotte-Rizzoli, P.: The predictability of large-scale wind-driven flows, Nonlin. Processes Geophys., 8, 449-465, 2001,

http://www.nonlin-processes-geophys.net/8/449/2001/.

Masuda, S., Akitomo K., and Awaji, T.: Effects stratification and bottom topography on the Kuroshio path variation south of Japan, I: Dependence of the path selection on velocity, J. Phys. Oceanogr., 29, 2419-2431, 1999.

Matsumoto, K. and Tsuda, I.: Noise-induced order, J. Stat. Phys., 31(1), 87-106, 1983.

McWilliams, J. C.: A note on a consistent quasi-geostrophic model in a multiply connected domain, Dyn. Atmos. Oceans, 1, 427441, 1977.

McWilliams, J. C. and Chow, J. H. S.: Equilibrium geostrophic turbulence I: A Reference Solution in a $\beta$-plane channel, J. Phys. Oceanogr., 11, 921-949,1981.

Nicolis, C.: Dynamics of model error: Some generic features, J. Atmos. Sci., 60, 2208-2218, 2003.

Nicolis, C.: Dynamics of model error: The role of unresolved scales revisited, J. Atmos. Sci., 61, 1740-1753, 2004.

Nicolis, C. and Nicolis, G.: Stochastic aspects of climatic transitions - additive fluctuations, Tellus, 33(3), 225-234, 1981.

Orrell, D., Smith, L., Barkmeijer, J., and Palmer, T. N.: Model error in weather forecasting, Nonlin. Processes Geophys., 8, 357-371, 2001 , http://www.nonlin-processes-geophys.net/8/357/2001/.

Pedlosky, J.: Geophysical Fluid Dynamics, Springer-Verlag, New York, 684 pp., 1987.

Perez-Munuzuri, V., Deza, R., Fraedrich, K., Kunz, T., and Lunkeit, F.: Coherence resonance in an atmospheric global circulation model, Phys. Rev., E71, 065602-1-065602-4, 2005.

Pikovsky, A. S. and Kurths, J.: Coherence resonance in a noisedriven excitable system, Phys. Rev. Lett., 78(5), 775-810, 1997.

Pikovsky, A., Rosenblum, M., and Kurtis, L.: Phase synchronization in regular and chaotic systems, Int. J. Bif. Chaos, 10, 22912305, 2000.

Pontryagin, L. S., Boltyanskii, V. G., Gamkrelidze, R. G., and Mishenko, E. F.: Mathematical Theory of Optimal Processes, Nauka, Moscow, 384 pp., 1969 (in Russian). (Also available as: Pontryagin, L. S., Boltyanskii, V. G., Gamkrelidze, R. G., and Mishenko, E. F.: The Mathematical Theory of Optimal Processes, The Mathematical Theory of Optimal Processes Authorized translation from the Russian by K. N. Trirogoff, Inter-
Science Publishers, New York, 360 pp., 1987).

Robinson, A. R., Arango, H., Warn-Varnas, G., Leslie, A. W. G., Miller, A. J., Haley, P. J., and Lozano, C. J.: Real-time regional forecasting, in Modern Approaches to Data Assimilation in Ocean Modeling, Elsevier Science, The Netherlands, 337409, 1996.

Rosenblum, M. G., Pikovsky, A. S., and Kurthis, J.: Synchronization approach to analysis of biological systems, Fluctuation and Noise Lett., 4(1), L53-L62, 2004.

Seki, K., Balakrishnan, V., and Nicolis, G.: Sensitivity to initial conditions in stochastic systems, Phys. Rev. E., 47(1), 155-163, 1993.

Stanev, E. V. and Staneva, J. V.: The impact of the baroclinic eddies and basin oscillations on the transitions between different quasistable states of the Black Sea circulation, J. Mar. Syst., 24, 3-26, 2000.

Stratonovich, R. L.: Topics in the Theory of Random Noise, Gordon and Breach, New York, Vol. 1, 324 pp., 1963.

Sura, P., Fraedrich, K., and Lunkeit, F.: Regime transitions in a stochastically forced double-gyre model, J. Phys. Oceanogr., 31, 411-426, 2001.

Toth, Z.: Estimation of atmospheric predictability by circulation analogs, Mon. Weather Rev., 125, 3297-3319, 1991.

Vannitsem, S.: The role of scale in the dynamics of parameterization uncertainties, J. Atmos. Sci., 63, 1659-1670, 2006.

Vannitsem, S. and Toth, Z.: Short-term dynamics of model errors, J. Atmos. Sci., 59, 2594-2603, 2002.

Vannitsem, S. and Nicolis, C.: Lyapunov vectors and error growth patterns in a T21L3 quasi-geostrophic model, J. Atmos. Sci., 54, 347-361, 1997.

Veronis, G.: Wind-driven ocean circulation, II: Numerical solutions of the nonlinear problem, Deep-Sea Res., 13, 31-55, 1966.

Von Storch, H. and Zwiers, F.: Statistical Analysis in Climate Research, Cambridge Univ. Press, Cambridge, New York, 494 pp., 1999.

White, H.: Estimation, Inference and Specification Analysis, Cambridge Univ. Press, Cambridge; New York, 382 pp., 1994.

Wirth, A. and Ghil, M.: Error evolution in the dynamics of an ocean general circulation model, Dyn. Atmos. Oceans, 32, 419-431, 2000.

Wright, P. B.: An atlas based on the COADS dataset: Fields of mean wind, cloudiness and humidity at the surface of global ocean, Report 14, Max-Plank-Institute für Meteorology, Hamburg, Germany, 1988.

Zelen, M. and Severo, N. C.: Probability functions, in: Handbook of Mathematical Functions with Formulas, Graphs, and Mathematical Tables, edited by: Abramowitz, M. and Stegun, I., Dover Publications, New York, 925-996, 1972. 